\title{
Space Charge in Polyethylene under AC Electric Stress using the Pulsed Electroacoustic Method
}

\author{
Y.L. Chong ${ }^{1}$, H. Miyake ${ }^{2}$, Y. Tanaka ${ }^{2}$, T. Takada ${ }^{2}$, G. Chen ${ }^{1}$ and H. Nakama ${ }^{2}$ \\ ${ }^{1}$ School of Electronics \& Computer Science, University of Southampton, UK \\ ${ }^{2}$ Electronics Measurement Laboratory, Musashi Institute of Technology, Tokyo, Japan
}

\begin{abstract}
This paper reports on space charge in XLPE planar samples of approximately $220 \mu \mathrm{m}$ subjected to dc $30 \mathrm{kV} / \mathrm{mm}$ and $1 \mathrm{~Hz}, 10 \mathrm{~Hz}$ and $50 \mathrm{~Hz}$ ac $30 \mathrm{kV}$ pk $/ \mathrm{mm}$ conditions for 24 hours. Measurement of space charge was carried out using the pulsed electroacoustic (PEA) technique. In addition, experiments with higher applied electric stress of $30 \mathrm{kV}_{\mathrm{rms}} / \mathrm{mm}$ and $60 \mathrm{kV}_{\mathrm{pk}} / \mathrm{mm}$ at $50 \mathrm{~Hz}$ ac condition were also conducted over an ageing period of 24 hours. In order to investigate the effect degassing on space charge distribution of samples under ac stress, both as-received and degassed were used for this study.
\end{abstract}

\section{Introduction}

Over the years, space charges within dielectrics under dc electric stress have received much interest and numerous literatures regarding their evolution and activities in different types of materials were published. On the other hand, space charge under $50 \mathrm{~Hz}$ ac condition has only received limited attention. Since electrical power is usually transmitted at $50 \mathrm{~Hz}$ ac, there is a cause for more investigations of space charge under such condition.

This paper reports on the result of space charge in both as-received and degassed Cross-linked polyethylene (XLPE) planar samples of approximately $220 \mu \mathrm{m}$ subjected to dc $30 \mathrm{kV}_{\mathrm{dc}} / \mathrm{mm}$ and ac (sinusoidal) electric stress level of $30 \mathrm{kV}_{\mathrm{pk}} / \mathrm{mm}$ at frequencies of 1 $\mathrm{Hz}, 10 \mathrm{~Hz}$ and $50 \mathrm{~Hz}$ ac for 24 hours.

In addition to these, ac space charge under 30 $\mathrm{kV}_{\mathrm{rms}} / \mathrm{mm}$ and $60 \mathrm{kV}_{\mathrm{pk}} / \mathrm{mm}$ electric stress at $50 \mathrm{~Hz}$ were also investigated.

\section{Automated PEA system}

The PEA is by far the most commonly used technique in the field of space charge measurement. This technique utilises the interaction between high voltage pulse and charge layers accumulated in the insulating material to produce acoustic pressure wave which transverse across the material. Detailed reviews on the principle of PEA can be found in numerous literature [1-2].

In dc space charge measurement, the applied voltage is not time dependent. However, for ac space charge measurement the applied voltage vary with time. It is therefore, important to correlate space charge with voltage. This is best achieved with the "point-onwave" method [3-4].

Figure 1 shows the schematic diagram of the PEA system developed by Musashi Institute of Technology in Japan.

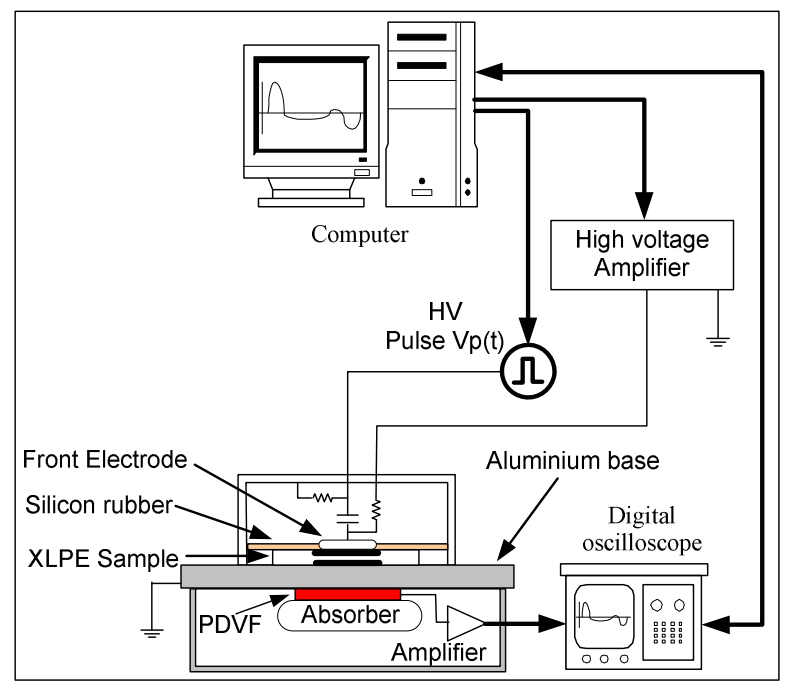

Figure 1. Schematic diagram of the pulsed electroacoustic technique

In this system, the computer can generate dc or ac sinusoidal waveform of various amplitude and frequencies. This waveform is then fed into a high voltage amplifier and finally across the sample. Since, the high voltage pulse required to generate the pressure wave is also controlled by the computer, therefore, it is possible to apply the high voltage pulses on the desired points of the ac waveform with precision to achieve the "point-on-wave" measurement approach. This is a different approach as reported in [3-4], which requires the voltage across the sample to be monitored by a capacitor divider.

\section{Sample details}

The test samples were approximately $220 \mu \mathrm{m}$ thick (bulk) made by the cable grade cross-linked polyethylene (Borealis XL4201S). Semicon electrodes were hot press into the plaque sample at $200{ }^{\circ} \mathrm{C}$ for 
about 10 minutes on both sides. The electrodes were made of the same grade of polyethylene material, but were loaded with carbon black to increase its conductivity (Borealis LEO592). Degassing was preformed on sample $\mathrm{G}$ to $\mathrm{L}$ to remove volatile byproducts of crosslinking agent. This was done in a vacuum oven at $90{ }^{\circ} \mathrm{C}$ for 48 hours.

\begin{tabular}{|c|c|c|c|}
\hline Sample & Treatment & Stress level & Frequency \\
\hline $\mathrm{A}$ & As-received & $30 \mathrm{kV}_{\mathrm{dc}} / \mathrm{mm}$ & $\mathrm{dc}$ \\
\hline $\mathrm{B}$ & As-received & $30 \mathrm{kV}_{\mathrm{pk}} / \mathrm{mm}$ & $1 \mathrm{~Hz}$ Sine \\
\hline $\mathrm{C}$ & As-received & $30 \mathrm{kV}_{\mathrm{pk}} / \mathrm{mm}$ & $10 \mathrm{~Hz}$ Sine \\
\hline $\mathrm{D}$ & As-received & $30 \mathrm{kV}_{\mathrm{pk}} / \mathrm{mm}$ & $50 \mathrm{~Hz}$ Sine \\
\hline $\mathrm{E}$ & As-received & $30 \mathrm{kV}_{\mathrm{rms}} / \mathrm{mm}$ & $50 \mathrm{~Hz}$ Sine \\
\hline $\mathrm{F}$ & As-received & $60 \mathrm{kV}_{\mathrm{pk}} / \mathrm{mm}$ & $50 \mathrm{~Hz}$ Sine \\
\hline $\mathrm{G}$ & Degassed & $30 \mathrm{kV}_{\mathrm{dc}} / \mathrm{mm}$ & $\mathrm{dc}$ \\
\hline $\mathrm{H}$ & Degassed & $30 \mathrm{kV}_{\mathrm{pk}} / \mathrm{mm}$ & $1 \mathrm{~Hz}$ Sine \\
\hline $\mathrm{I}$ & Degassed & $30 \mathrm{kV}_{\mathrm{pk}} / \mathrm{mm}$ & $10 \mathrm{~Hz}$ Sine \\
\hline $\mathrm{J}$ & Degassed & $30 \mathrm{kV}_{\mathrm{pk}} / \mathrm{mm}$ & $50 \mathrm{~Hz}$ Sine \\
\hline $\mathrm{K}$ & Degassed & $30 \mathrm{kV}_{\mathrm{rms}} / \mathrm{mm}$ & $50 \mathrm{~Hz}$ Sine \\
\hline $\mathrm{L}$ & Degassed & $60 \mathrm{kV}_{\mathrm{pk}} / \mathrm{mm}$ & $50 \mathrm{~Hz}$ Sine \\
\hline
\end{tabular}

Table 1. Sample details

\section{Experimental protocols}

All samples were aged for a duration of 24 hours. Space charge distributions were measured at every hour interval during ageing period with voltage applied.

The "point-on-wave" approach is not necessary for dc space charge since the applied voltage does not vary with time. For the case of ac space charge, however, 16 equally spaced measurements over a complete sinusoidal wave were taken.

After 24 hours of ageing, the short-circuit space charge distribution was also measured.

\section{Results and discussion}

Figure 2a shows the space charge profiles during ageing at 0 hour and 24 hours. At 0 hour, slight amount of homocharges were accumulated at both electrodes. At 24 hours, a significant amount of positive charge (heterocharge) starts to accumulate at the cathode with slight amount of negative charge accumulating at the anode. This is probably due to ionic dissociation of byproducts in the XLPE sample.
With reference to figure $2 \mathrm{~b}$, significant amount of positive charge is being trapped at the vicinity of the cathode when short-circuited after 24 hours of ageing. The voltage off space charge profile also shows slight accumulation of positive homocharge near the anode. A layer of negative charge is sandwiched between the positive charges at the anode and cathode. The accumulated charge was observed to reduce after shortcircuiting for the sample for 2 hours.
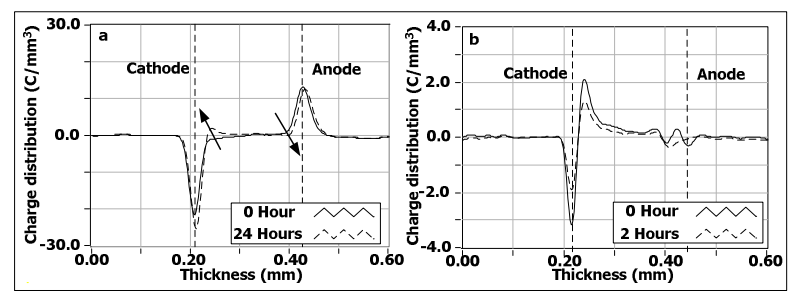

Figure 2. Charge profiles of sample A: (a) aging (b) short-circuit

The space charge profiles of sample B at phase $90^{\circ}$ and $270^{\circ}$, which correspond to $30 \mathrm{kV}_{\mathrm{pk}} / \mathrm{mm}$ and -30 $\mathrm{kV}_{\mathrm{pk}} / \mathrm{mm}$ are shown in figure $3 \mathrm{a}$ and $3 \mathrm{~b}$ respectively. There appears to be a very small amount of heterocharges near the vicinity of electrodes when the phase voltage is $30 \mathrm{kV}_{\mathrm{pk}} / \mathrm{mm}$. This heterocharges become homocharges to the electrodes during the $270^{\circ}$.
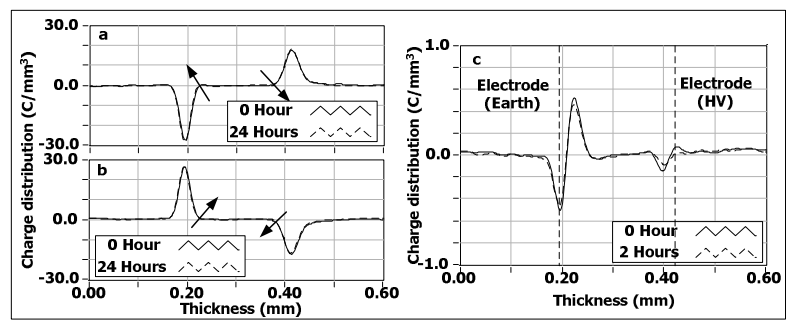

Figure 3. Charge profiles of sample B: (a) at phase of $90^{\circ}$ (b) at phase of $270^{\circ}$ (c) short-circuit

The short-circuit space charge profile of sample B is dominated by positive charge accumulation near the earth electrode while a small amount of negative charge can be seen at the HV electrode. An even smaller but opposite charge can be observed between these two layer of charge. This maybe due to charge carriers with lower motilities than the ones trapped near the two electrodes. As in sample A, charge reduction can be observed after short circuiting the sample for 2 hours.

Figure 4 shows the short-circuit space charge profiles of samples $\mathrm{C}$ to $\mathrm{F}$. The space charge profile of sample $\mathrm{C}$ appears somewhat similar to that of sample $\mathrm{B}$, except with the notable difference of the charge accumulated at the vicinity of the electrodes reduces while the charge in the bulk increases. This is probably because at $10 \mathrm{~Hz}$, the frequency at which the polarity 
voltage varies is too fast for charge to be trapped near the electrodes and hence remains in the middle.

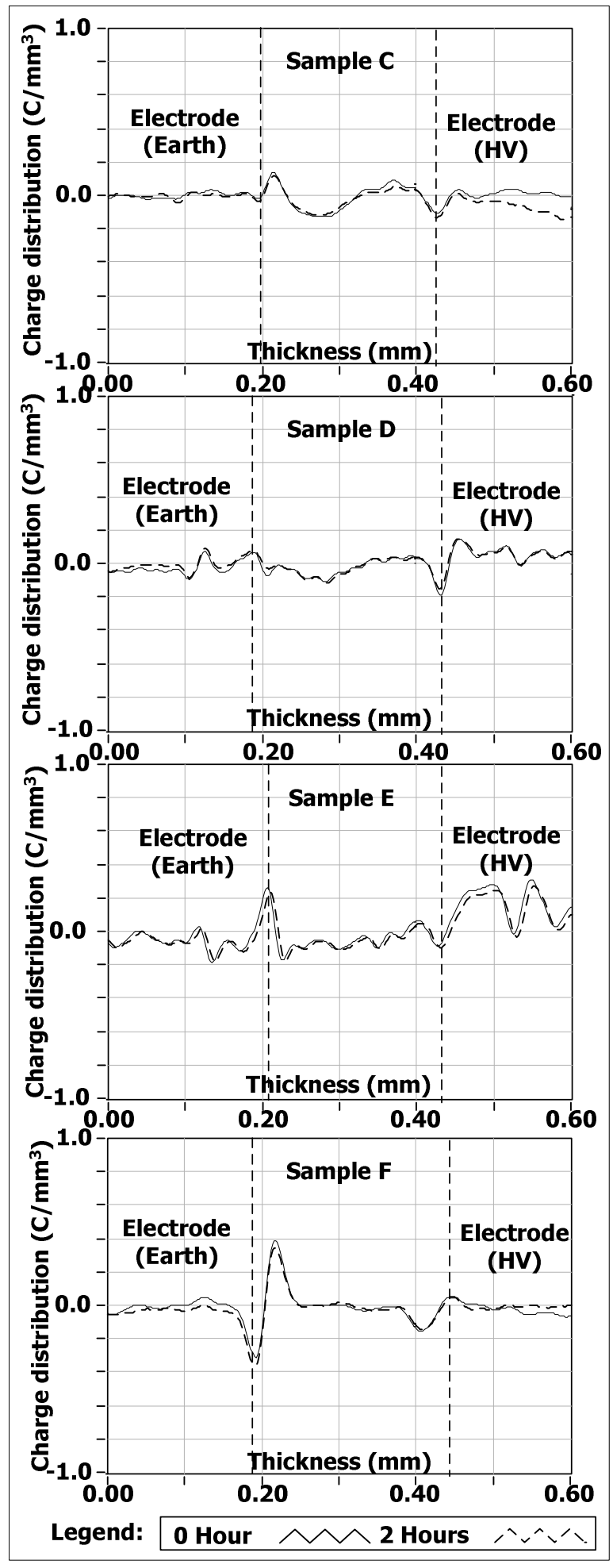

Figure 4. Short-circuit charge profiles of as-received samples.
In the case of sample $\mathrm{D}$, the voltage variation (50 $\mathrm{Hz}$ ) is even greater and hence the amount of charge trapped is even smaller than that seen in sample $\mathrm{C}$.

Increasing the applied stress to $30 \mathrm{kV}_{\mathrm{rms}} / \mathrm{mm}$ results in more negative charge accumulation in the bulk as seen in sample E. While further increasing to the stress level to $60 \mathrm{kV}_{\mathrm{pk}-\mathrm{pk}} / \mathrm{mm}$ result in space charge distribution that resembles that of which corresponds to dc stress. This is can be clearly seen when comparing the short circuit space charge distribution of samples A and $F$.

One possible explanation for this is that at $50 \mathrm{~Hz}$ ac stress the alternating voltage is constantly charging and discharging the sample. Hence it takes a much higher stress level to accumulate the kind of space charge it takes for a sample under lower dc stress to accumulate.

It is note worthy that for samples D to F, short circuiting the samples for 2 hours produced little reduction in accumulated charge. This maybe due to formation of deep traps caused by ac ageing.

Homocharges were accumulated near the vicinity of the electrodes of sample $\mathrm{G}$ as shown in figure 5. This accumulation of homocharges is most probably due to charge injected direct from the electrodes. Short circuiting the sample for two hours result in reduction of charge, particularly near the earth electrode although not as much as sample A.

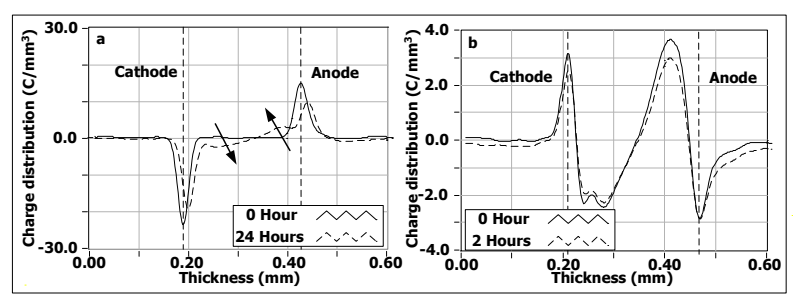

Figure 5. Charge profiles of sample G: (a) aging (b) short-circuit

Referring to figure 6 , it can be seen that, with the exception of sample I, which shows no charge, negative charge is accumulated at both ends of the electrodes for all other samples.

In the case of sample $\mathrm{H}$, there is higher probability for charge injected from the electrodes to be trapped due to the relatively longer time lag before the voltage changes polarity. On the other hand, at a frequency of $10 \mathrm{~Hz}$, the voltage varies too fast for any noticeable charge to be trapped in sample I. One would expect no charge accumulation when the frequency of applied voltage is $50 \mathrm{~Hz}$. However, a quick look at the space charge profiles of sample J suggest otherwise. 


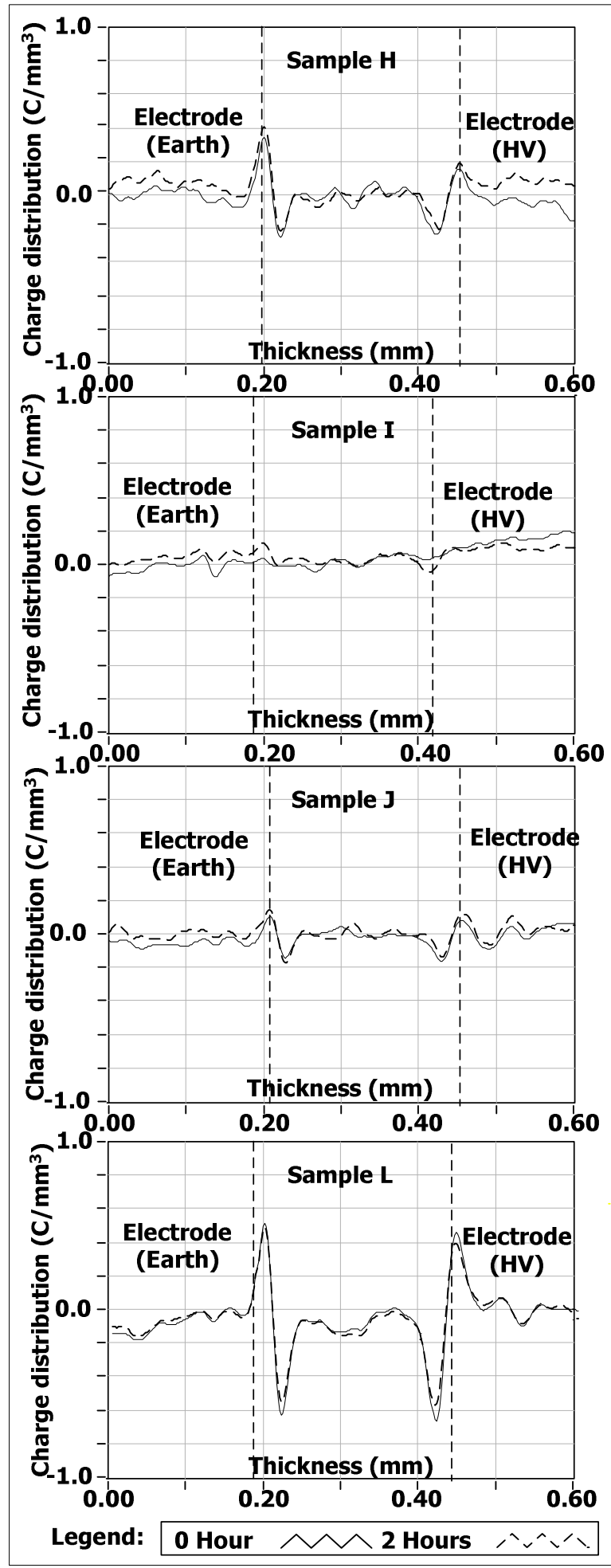

Figure 6. Short-circuit charge profiles of degassed samples

One possible explanation for this is perhaps at 50 $\mathrm{Hz}$, sample $\mathrm{J}$ was subjected to more cycles of polarity reversal for the given ageing duration as compared to both samples $\mathrm{H}$ and $\mathrm{I}$. This may result in more rapid degradation and/or formation of deeper traps.

Comparing the space charge profiles of samples $\mathbf{J}$ and $\mathrm{L}$ indicate an increase in the accumulated charge. This maybe attributed to the fact that higher electric stress enhances charge injection and the process describe before. The space charge profile of sample $\mathrm{K}$ is somewhere between sample $\mathbf{J}$ and $\mathrm{L}$ and hence will not be shown in this paper.

Relatively less decay in accumulated charge was observed in samples $\mathrm{H}, \mathrm{J}$ and $\mathrm{L}$ as compared to samples $\mathrm{G}$ after short circuiting the samples for 2 hours. This further supports the earlier statement that ac ageing results in formation of deeper traps.

\section{Conclusion}

Space charge in XLPE planar samples, both as-received and degassed, subjected to dc and ac electric stress was reported.

Accumulated space charge, albeit very small amount, can be seen in most of the samples tested. For as-received samples, the amount of accumulated charge was found to reduce with increasing frequency of the applied voltage.

In the case of degassed sample, no significant charge can be observed in the sample subjected to $10 \mathrm{~Hz}$ ac electric stress, while charge was observed at frequencies of $1 \mathrm{~Hz}$ and $50 \mathrm{~Hz}$. It is believed that a combination of charge injection, trapping, formation of deep traps and material degradation caused by ac ageing is accountable for this phenomena.

\section{Acknowledgement}

The authors would like to thank the Ministry of Education, Culture, Sports, Science and Technology of Japan for funding this student exchange program.

\section{References}

[1] T. Maeno, T. Futami, H. Kushibe, T. Takada and C.M. Cooke, "Measurement of spatial charge distribution in thick dielectrics using the pulsed electroacoustic method," IEEE Trans. on EI. Vol 23. pp.433-439,1983.

[2] P. Morshuis and M. Jeroense, "Space charge measurements on impregnated paper: A review of the PEA method and a discussion of results," IEEE EI. Mag.pp.26-35, 1997.

[3] Y. F. F. Ho, G. Chen, A. E. Davies, R. N. Hampton, S. Hobdell, S. G. Swingler and S. J. Sutton, " Space Charge Measurements In XLPE insulation under $50 \mathrm{~Hz}$ ac electric stress," DMMA, pp 68-73, 2000.

[4] Y. F. F. Ho, G. Chen, A. E. Davies, S. G. Swingler, S. J. Sutton, R. N. Hampton and S. Hobdell " Measurement of space charge in XLPE insulation under $50 \mathrm{~Hz}$ ac electric stresses using the LIPP method,"IEEE Trans. DEI vol. 9. pp.362-370, 2002. 